\title{
INCREASES IN SCR ENERGETIC PROTON FLUXES ON EARTH AND THEIR RELATION TO SOLAR SOURCES
}

\author{
L.L. Lazutin \\ Skobeltsyn Institute of Nuclear Physics MSU, \\ Moscow,Russia,lll@srd.sinp.msu.ru
}

\begin{abstract}
Logachev catalog data for solar cycle 23 has been used to study the dependence of measured increases in solar cosmic rays (SCRs) on solar perturbations. The efficiency of recording the SCR increases, driven by proton acceleration in the corona, on Earth and in its vicinity is shown to depend on power of a solar flare that created a shock wave and on position of the flare on the solar disk. As the particle flux moves along the heliolongitude away from the parent flare, the acceleration efficiency decreases, i.e. the maximum
\end{abstract}

energy of the accelerated particles and their intensity at equal energy decrease. As a result, at a certain distance along a heliolongitude from the parent solar flare, the solar proton flux intensity decreases to the galactic background, and there is no SCR increase detected.

Keywords: solar cosmic rays, solar flare intensity, heliolongitude.

\section{INTRODUCTION}

Solar cosmic rays (SCR) were discovered as early as the last century and since then have been well studied using measurements on Earth, in the stratosphere, and in interplanetary space. Over the years, a large number of theoretical models of particle flux acceleration and prognostic systems necessary for practical activity in interplanetary space have been developed. Proton flares are generally divided into two groups - short and long (see the review [Desai, Giacalone, 2016] and references therein). This paper mainly deals with prolonged increases in energetic proton fluxes generated, according to [Desai, Giacalone, 2016], by shock wave acceleration in the solar corona.

Detection of SCR on Earth and its orbit (hereinafter referred to as "on Earth" " for short) is determined by the location and the mechanism for generating particles on the solar disk and their propagation from the Sun to Earth.

To date, two mechanisms for SCR generation on the Sun and one in interplanetary space have been proposed; each observed in measurements: acceleration directly during an optical flare in the flare region (socalled flare acceleration) and acceleration of a shock in the corona (hereinafter, coronal acceleration), as well as in interplanetary space (hereinafter, interplanetary acceleration). The SCR enhancements caused by these acceleration mechanisms are further referred to as flare, coronal, and interplanetary as well. This paper does not describe interplanetary acceleration since it is usually limited to lower energies.

Flare acceleration leads to an SCR enhancement that is detected on Earth only when the flare is at solar longitudes $60-80^{\circ} \mathrm{W}$, from which magnetic field lines in interplanetary space come helically directly to Earth, i.e. in the interval of geoeffective heliolongitudes. The SCR enhancements induced by flares at other heliolongitudes are not recorded on Earth because the transfer of protons across magnetic field lines, in interplanetary space, and in the solar corona is impeded [Ellison, Ramaty,
1985; Reames, 1999, 2013]. For this reason, the contribution of solar flare particles to the total number of ground level SCR enhancements is small.

Flare acceleration occurs deeply enough in the solar photosphere, and in this case the composition of accelerated particles, due to the high matter density in the acceleration region, differs from that during coronal and interplanetary accelerations. Nonetheless, we can distinguish the flare SCR enhancement from the enhancement caused by coronal mass ejections (CMEs) at west heliolongitudes only by the depth of acceleration and by the SCR nuclear composition, which requires individual analysis of each enhancement.

For example, Kurt et al. [2019] have examined pion fluxes generated by solar protons in a flare with west coordinates on an interval of geoeffective heliolongitudes on September 10, 2017. Pions are formed by protons in fairly deep solar layers, and the time coincidence of proton and pion fluxes indicates that SCRs have flare origin.

Coronal SCR enhancements are linked to the particle flux acceleration by a CME shock in the solar corona. The shock spans a large part of solar longitudes, and the accelerated particle flux goes into interplanetary space also at a distance in heliolongitude from the parent flare. On Earth, the coronal SRC enhancements are, therefore, recorded more often than the flare ones.

The present work focuses on the coronal SCR enhancements. It should be noted that the question about dependence of geoeffectiveness of solar flares on the heliolongitude of an active region has been raised earlier by V.P. Maksimov with co-authors. They obtained a patent for an invention of a method of predicting strong solar flares [Maksimov et al., 1996]. The problem remained relevant since the relationship of ground-based observations of SCRs with the position and power of the parent solar flare was not studied.

Shock-wave acceleration in interplanetary space is limited by the low-energy portion of the spectrum since solar wind particles are accelerated, as opposed to coro- 
nal acceleration at which the initial proton energy is much higher. In fact, if flares occur often, there may also be more energetic particles in interplanetary space, but such complex cases require individual consideration.

Since interplanetary acceleration is usually limited to low energy, and flare acceleration is recorded on limited field lines adjacent to Earth, most of the increases in energetic solar proton fluxes observed on Earth owe their origin precisely to coronal acceleration. This opinion has also been expressed in most recent works [Ellison, Ramaty, 1985; Reames, 1999, 2013].

As initial data we have used solar proton events (SPEs) of solar cycle 23 (1998-2007), collected in the catalog created by Yu.I. Logachev [Logachev et al., 2014a]. This and other catalogs by Logachev have been discussed in several papers [Kudela, Lazutin, 2011; Logachev et al., 2014b, 2015; Bazilevskaya et al., 2015; Miroshnichenko, 2018]. In this paper, we study features of the connection between SCR enhancements recorded on Earth and the position and power of the optical solar flares responsible for the enhancements. The cases when we cannot accurately identify a flare and its related CME shock are omitted. We mainly utilize the ROES satellite measurements in polar caps that enable us to construct the time variation of proton fluxes and to assess their spectral characteristics due to a large number of energy channels.

The purpose of this work is to find the dependence of the maximum energy and intensity of coronal SCR enhancements recorded on Earth on the power of solar events and their positions on the solar disk.

\section{MEASUREMENT RESULTS}

Figure 1 gives an example of the development of increasing SCR proton fluxes on November 08-10, 2000.

The optical flare responsible for this enhancement occurred at a heliolongitude of $77^{\circ} \mathrm{W}$ on the interval of geoeffective heliolongitudes. This reflected in the steep front of increasing proton fluxes. The coronal energetic particle flux, as the area of propagation in interplanetary space extends, adiabatically decreases with time, and this is also shown in Figure 1.

The enhancement in the low-energy particle flux is caused by the shock-wave acceleration in interplanetary space and its interaction with the magnetosphere. At high energies (over $16 \mathrm{MeV}$ ), there are no proton flux enhancements in interplanetary space because the initial energy of solar wind particles is low and during the shock wave motion to Earth's orbit no acceleration to high energies is detected. This feature is reflected in the change of the energy spectrum of particles shown in Figure 2 for three parts of the time variation of the proton increase.

Figure 3 plots the number of SCR enhancements detected on Earth as a function of the heliolongitudinal position of respective solar flares. Obviously, there are only a handful of cases of detection of SCR protons from the eastern-most flares, although, in theory, the heliolongitude distribution of solar flares should be uniform. The observed pattern can be attributed to a

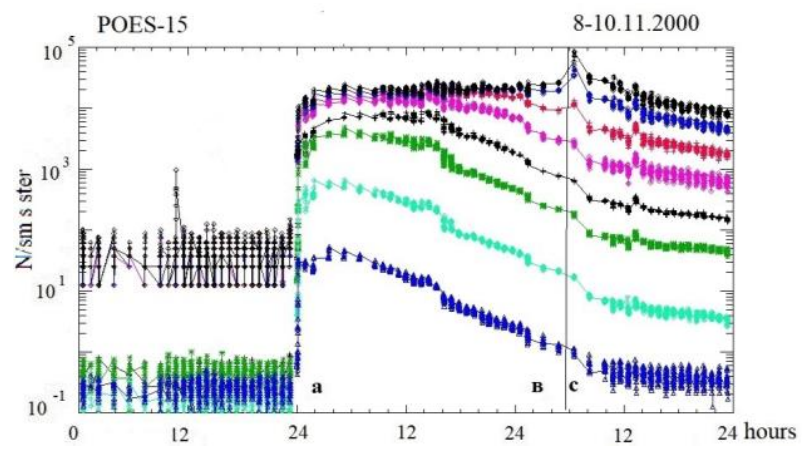

Figure 1. Proton fluxes measured by the POES-15 satellite in the polar cap in the November 08, 2000 SCR flare: from the bottom upwards - energies greater than 140, 70, 36, 16, 6.9, 2.5, 0.8 , and $0.25 \mathrm{MeV}$

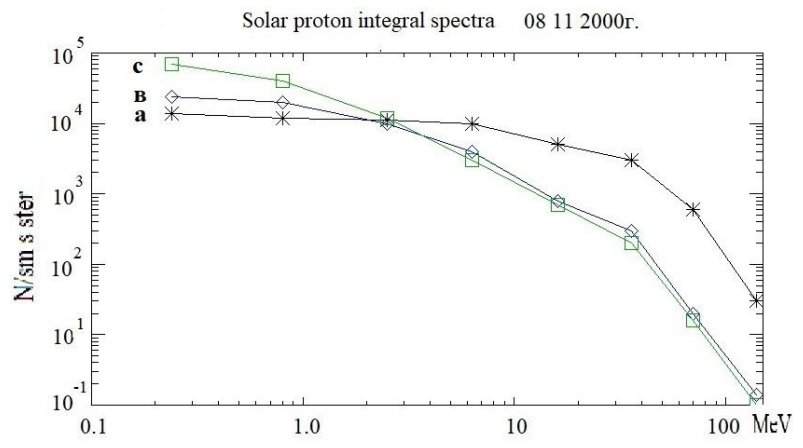

Figure 2. Integral SCR proton spectra measured at the moments indicated in Figure 1 in the November 08-10, 2000 flare

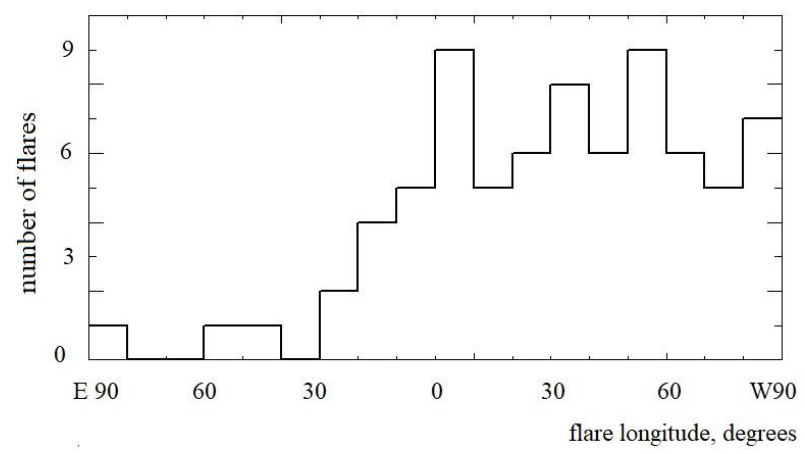

Figure 3. Heliolongitude distribution of solar flares responsible for SPEs from Logachev's catalog. The distribution is similar to those obtained in [Reames, 1999, Desai, Giacalone, 2016]

decrease in the acceleration efficiency with distance in longitude away from the maximum, i.e. from the longitude of a solar flare.

Let us consider how the accelerated proton flux depends on flare longitude and importance. To this end, Figure 4 shows all cases of ground level and near-Earth SCR enhancements as function of heliolongitude and importance of the initial solar flare. A few cases have been excluded from consideration in which the identification was not positive or there was a significant impact of adjacent flare SCR enhancements.

The resulting dependence can be understood if we assume that the flux and energy of accelerated protons decrease with distance in longitude away from the parent flare. The higher is the flare importance, the farther 


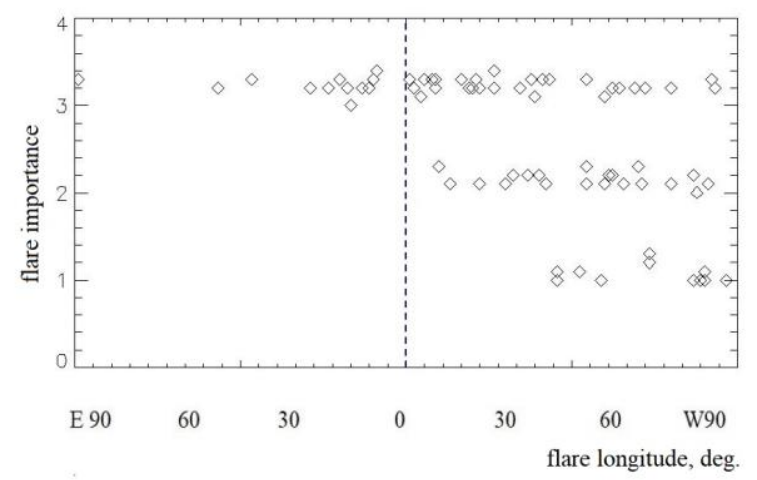

Figure 4. Coronal SCR enhancements on Earth as function of flare heliolongitude and importance

from it in longitude a coronal accelerated proton flux is visible on Earth. Thus, the proton fluxes accelerated by a shock in the corona as a result of a flare of importance 3 will cause a ground level SCR enhancement at almost any position of the parent flare. The decrease in the number of flares at east $\left(0^{\circ}-40^{\circ}\right)$ heliolongitudes can be explained by the fact that the optical importance only roughly reflects the acceleration efficiency of SCR fluxes. A flare of importance $2(\mathrm{~N})$ leads to a ground level SCR enhancement only if it occurs at west heliolongitudes; those solar particle fluxes that are accelerated at east heliolongitudes lose velocity at the terrestrial part of the Parker spiral, decreasing to the level of background galactic cosmic rays; hence, no ground level SCR enhancement is detected. Accordingly, weak flares of importance 1 (F) generate a shock able to cause a ground level SCR enhancement only if they occur to the west of the $50^{\circ}$ heliolongitude; those observed to the east of the geoeffective heliolongitude interval produce a proton flux, which, when approaching Earth, decreases to the level of the galactic background.

There is no doubt that the optical flare importance is not an accurate indicator of the particle acceleration efficiency, but the dependence of ground-based detection of SCR enhancement on the position and power of the parent flare and its related shock in the solar corona, which follows from Figure 3, is in little doubt. In our opinion, to find this dependence it suffices to have data on one solar cycle from Logachev's catalog.

\subsection{Flare acceleration}

Flare acceleration is the earliest explanation for SCR enhancements, which has long been the only one. Yet, during direct propagation, the proton fluxes detected on Earth are accelerated in flares located at west heliolongitudes, where magnetic field lines in interplanetary space are connected with Earth by the Parker spiral. According to present-day views, protons accelerated by flares at other heliolongitudes cannot reach Earth because the drift across magnetic field lines is much less effective. This statement also applies to the transfer of protons in the solar corona - we can see this in Figure 4 where half of flares of importance 1 and 2, which occurred at east heliolongitudes, did not cause ground level SCR enhancements.
Thus, flare acceleration can explain only a handful of ground level SCR enhancements, when solar flares occur in the close vicinity of the geoeffective heliolongitude interval. To explain the relationship between SCR enhancements recorded on Earth and solar activity effects, it is necessary to invoke another acceleration mechanism, namely the acceleration of particles by shocks in the corona.

\subsection{Coronal acceleration}

Coronal particle acceleration coincides in time with solar flares and CMEs. Particle fluxes in the solar corona are accelerated at the CME shock front up to energies of tens of $\mathrm{GeV}$. This mechanism has been examined by both Russian [Shabansky, 1961; Krymskii, 1977; Berezhko et al., 1988; Berezhko, Taneev, 2013] and foreign [Axford et al., 1977; Ellison, Ramaty, 1985; Reames, 1999, 2013] authors. In these works, most SCR enhancements are attributed to the acceleration of SCR proton fluxes by a shock moving to Earth ahead of its driven CME. The initial particle flux may include particles accelerated not only by the flare responsible for this CME, but also by the previous adjacent flare.

In the intermediate position, when parent solar flares are near the central meridian, characteristics of proton flux enhancements are also intermediate: the ground level enhancement is shorter than that during east flares, but its profile is not so steep as in west flares. Note that in many cases, the time variation in SCR fluxes is not so simple: a combination of two or more flares complicates the picture.

Solar flares themselves should be distributed uniformly over latitude, but Figure 4 demonstrates a clear shortage of proton flares at east heliolongitudes. We may suppose that a large part of solar flares at east heliolongitudes produces CMEs that on their west wing lose their ability to accelerate SCR fluxes. This is most likely due to a decrease in the shock wave velocity with distance from the epicenter of the flare. Moreover, Figure 4 confirms the conclusion about the absence of perpendicular propagation of SCRs both in the corona and in interplanetary space.

The lower is the flare importance, the closer to west heliolongitudes should propagate the shock-accelerated particle fluxes to cause a visible ground level SCR enhancement. To the east of the central meridian, there are no geoeffective flares of importance 1 and 2 (see Figure 4) since weak flares at east heliolongitudes generate CMEs and particle fluxes shorter in longitude, and even the fluxes they accelerate to energies above the galactic background do not reach west heliolongitudes magnetoconjugate with Earth.

Accordingly, it is easy to explain the duration distribution of SCR enhancements. The longest SCR enhancements are caused by strong flares at east heliolongitudes; the shortest, by weak flares at west heliolongitudes. Thus, we have built a continuous series of durations of solar proton events - from the longest to the shortest, and the common division into two categories proves to be incorrect. Note, however, that it is a simpli- 
fication to suspect that the particle flux enhancement intensity depends only on the flare optical importance, but it allows us to find new laws.

\section{CONCLUSIONS}

We have studied two mechanisms of energetic SCR flux acceleration - flare and coronal. The traditional flare mechanism works in a narrow sector of west heliolongitudes, around the geoeffective heliolongitude interval - the region where magnetic field lines are connected to Earth. According to the coronal mechanism, most SCR flares are attributed to shock wave acceleration of particle fluxes in the solar corona.

The main conclusion of this work is that the ground level SCR enhancement and its intensity depend on the combination of power and heliolongitude of a solar flare that produced the shock. The closer is the flare to the geoeffective heliolongitude interval and to the point of intersection of the magnetic field line with Earth, the more likely is that the flux of protons accelerated by the shock in the corona has no time to decrease to the galactic background level and is detected by ground-based instruments. Near the point of intersection, the acceleration by a shock generated by both weak and strong flares proves to be effective, but the more marked is the shift in heliolongitude to east, the greater should be the flare importance so that it can produce a proton flux perceptible on Earth. Therefore, only flares of importance 3 with the east heliolongitude can cause SCR enhancements detectable on Earth. Of course, the optical flare importance only roughly reflects the efficiency of generation of protons, but, in our opinion, this does not affect the correctness of the pattern shown in Figure 3. Using the time interval that spans more than one 11year cycle can improve the accuracy of research, but does not change the meaning of this pattern.

Thus, proton fluxes accelerated in solar flares can be recorded on Earth if a flare occurs at west heliolongitudes near field lines adjacent to Earth. Most ground level SCR enhancements are due to proton flux acceleration by a CME shock in the solar corona, the acceleration efficiency (and, hence, the energy and intensity of the proton flux) is higher the closer is the parent flare to magnetoconjugate point and the higher is the flare importance. As a result, at east heliolongitudes only flares of high importance can induce the SRC enhancements detectable on Earth, and the flares of the lowest importance should be near the magnetoconjugate point. i.e. as close to the interval of west geoeffective heliolongitudes as possible so that SCR fluxes from them can reach Earth.

The work was supported by RFBR grant No. 19-0200264 .

\section{REFERENCES}

Axford W.I., Leer E., Skadron G. The acceleration of cosmic rays by shock waves. $15^{\text {th }}$ Intern. Cosmic Ray Conf., Plovdiv, Bulgaria. 1977, vol. 11, pp. 132-137.

Bazilevskaya G.A., Logachev Y.I., Daibog E.I., Lazutin L.L., Miroshnichenko L.I., Surova G.M., Yakovchouk O.S., Vashenyuk E.V., Ishkov V.N., Nazarova M.N., Petrenko I.E. Solar proton events in solar activity cycles 21-24. Bulletin of the Russian Academy of Sciences: Physics. 2015, vol. 79, no. 5, pp. 573-576. DOI: 10.3103/S1062873815050135.

Berezhko E.G., Elshin V.K., Krymskii G.F., Petukhov S.N.
Generatsiya kosmicheskikh luchei udarnymi volnami [The generation of cosmic rays in shock waves]. Novosibirsk, Nauka Publ., 1988, 189 p. (In Russian).

Berezhko E.G., Taneev S.N. Shock acceleration of solar cosmic rays. Astron. Lett. 2013, vol. 39, no. 6, pp. 393-403. DOI: $10.1134 / \mathrm{S} 1063773713060017$.

Desai M., Giacalone J. Large gradual solar energetic particle events. Living Rev. Solar Phys. 2016, vol. 13, 3. DOI: $10.1007 / \mathrm{s} 41116-016-0002-5$.

Ellison D.C., Ramaty R. Shock acceleration of electrons and ions in solar flares. Astrophys. J. 1985, vol. 298, pp. 400-408.

Krymskii G.F. A regular mechanism for the acceleration of charged particles on the front of a shock wave. Soviet Phys. Dokl. 1977, vol. 22, pp. 327-328.

Kudela K., Lazutin L. Selected solar influences on the magnetosphere: Information from cosmic rays. The Sun, the Solar Wind, and the Heliosphere. Proceedings of the conference held 23-30 August, 2009 in Sopron, Hungary. Berlin, Springer, 2011, pp. 199-207. (IAGA Special Sopron Book Series, vol. 4.). DOI: 10.1007/978-90-481-9787-3 18.

Kurt V., Belov A., Kudela K., Mavromichalaki H., Kashapova L., Yushkov B., Sgouropoulos C. Onset time of the GLE 72 observed at neutron monitors and its relation to electromagnetic emissions. Solar Phys. 2019, vol. 294, no. 2, 22. DOI: 10.1007/s11207-019-1407-9.

Logachev Yu.I., Bazilevskaya G.A., Vashenyuk E.V., Daibog E.I., Ishkov V.N., Lazutin L.L., Miroshnichenko L.I., Nazarova M.N., Petrenko I.E., Stupishin A.G., Surova G.M., Yakovchuk O.S. Catalogue of solar proton events of 19972009. Edited by Yu.I. Logachev. 2014a. http://www.wdcb.ru/ stp/stp.ru/catCDR_r (accessed July 15, 2020).

Logachev Y.I., Bazilevskaya G.A., Vashenyuk E.V., Daibog E.I., Ishkov V.N., Lazutin L.L., Miroshnichenko L.I., Nazarova M.N., Petrenko I.E., Stupishin A.G., Surova G.M., Yakovchouk O.S. Catalogues of solar proton events and their sources in solar cycles 20-23. Kosmicheskie luchi $i$ solnechnaya aktivnost' [Cosmic Rays and Solar Activity]. Moscow, Russian Academy of Natural History, International Academy of Appraisal and Consulting, 2014b, pp. 155-175. (Ser. «Cosmic Rays», vol. 29). (In Russian).

Logachev Y.I., Daibog E.I., Lazutin L.L., Miroshnichenko L.I., Surova G.M., Yakovchouk O.S., Bazilevskaya G.A., Vashenyuk E.V., Ishkov V.N., Nazarova M.N., Petrenko I.E., Stupishin A.G. A comparison of proton activity in cycles 2023. Geomagnetism and Aeronomy. 2015, vol. 55, no. 3, pp. 277-286. DOI: 10.1134/S0016793215030135.

Maksimov V.P., Bakunina V.P., Bakunina I.A., Nefedyev V.P., Smolkov G.Ya. Method of short-term forecast of powerful solar flares. Patent RU 2114449 C1. 1996. https://yandex.ru/patents/doc/RU2114449C1_19980627 (accessed July 15, 2020).

Miroshnichenko L.I. Solar cosmic rays: 75 years of research. Physics-Uspekhi. 2018, vol. 61, no. 4, pp. 323-352. DOI: $10.3367 /$ UFNe.2017.03.038091.

Reames D.V. Particle acceleration at the Sun and in the heliosphere. Space Sci. Rev. 1999, vol. 90, pp. 413-491.

Reames D.V. The two sources of solar energetic particles. Space Sci. Rev. 2013, vol. 175, iss. 1-4, pp. 53-92. DOI: $10.1007 / \mathrm{s} 11214-013-9958-9$.

Shabansky V. P. Particle acceleration by passage of a hydromagnetic wave front. Zhurnal eksperimental'noi i teoreticheskoi fiziki [JETP]. 1961, vol. 41, no. 4, pp. 1107-1111. (In Russian). (English edition: Shabanskii V.P. Particle acceleration by passage of a hydromagnetic wave front. JETP. 1962, vol. 14, no. 4, pp. 791-793).

How to cite this article

Lazutin L.L. Increases in SCR energetic proton fluxes on Earth and their relation to solar sources. Solar-Terrestrial Physics. 2020. Vol. 6. Iss. 4. P. 40-43. DOI: $10.12737 /$ stp-64202006. 